\title{
Zur Kenntniss der Trypsinwirkung.
}

\author{
Von
}

A. Kossel und A: Mathews.

An die in der vorhergehenden Abhandlung niedergelegten Versuchsergebnisse, weiche den chemischen Bau der Protamine in gröberem Umriss feststellen und ihre Analogie mit den Eiweisskörpern einerseits, mit den Polysacchariden andererseits erweisen, knüpft sich die Frage nach dem Verhalten dieser Stoffe $z u$ den Fermenten. Wir haben die Wirkung des Pepsins und des Trypsins zu dem Protamin geprüft und theilen die vorläufigen Resultate dieser Versuche im Folgenden mit.

Zunächst wurden Versuche über die Einwirkung des Pepsins auf Salmin angestellt. Hierbei zeigte sich, dass die eiweissfällende Wirkung der Protaminlösung selbst nach langdauernder kräftiger Pepsinverdauung noch erhalten ist. Da die nächsten peptonartigen Spaltungsprodukte der Protamine, die Protone, kein Eiweiss fällen, so muss man hieraus den Schluss ziehen, dass das Protamin unzersetzt geblieben ist. Auch die übrigen Reactionen des Protamins zeigten nach der Pepsinwirkung keine Veränderung.

Eine tiefgreifende Wirkung übt das Trypsin aus. Wenn man eine Lösung von Protamin mit einer nach Kühne und Chittenden bereiteten Trypsinflüssigkeit ${ }^{1}$ ) unter Zusatz von Chloroform im Brütofen digerirt, so beobachtet man ziemlich bald eine Veränderung der Reactionen. Die Biuretreaction, welche Anfangs eine blauviolette Farbe ergibt, wird mehr

1) Zeitschrift für Biologie. 1885, Bd. 22, S. 428. 
roth, die eiweissfällende Wirkung verschwindet. Auch die Reaction mit wolframsaurem Natron gibt über die Umwandlung des Protamins Aufschluss. Wie in der vorhergehenden Abhandlung bemerkt, giebt das Protamin selbst bei schwach alkalischer Reaction mit diesem Reagens eine Fällung, die Protone hingegen geben eine solche Fällung nicht bei alkalischer, sondern nur bei saurer Reaction der Flüssigkeit. Im Laufe der Trypsinverdauung des Protamins tritt diese Reaction Anfangs sowohl bei saurer wie bei alkalischer, später nur bei saurer Reaction, endlich überhaupt nicht mehr ein. Ebenso verschwindet auch die Fällbarkeit durch Ferrocyankalium (und Essigsäure) ziemlich bald.

Die Verdauung . wurde in den unten beschriebenen Versuchen gewöhnlich soweit fortgesetzt, bis die Biuretreaction in der verdauten Protaminlösung annähernd ebenso ausfiel, wie in einer ohne Protamin angesetzten Kontrollprobe des Pankreasextracts.

Aus einer grösseren Zahl derartiger Versuche ergab sich, dass die Protone unter dem Einfluss des Trypsins in Hexonbasen zerlegt werden. Ausserdem kann aus der Lösung ein krystallisirender Körper gewonnen werden, der wahrscheinlich als ein Zwischenprodukt zwischen Protonen und Hexonen aufzufassen ist. Kontrollversuche lehrten, dass die Trypsinlösung allein nur einen kleinen Bruchtheil der überhaupt entstehenden Basen liefern konnte.

Da die Protone bisher überhaupt nicht genauer charakterisirt worden sind, so haben wir auf ihre Darstellung aus den Produkten der Trypsinverdauung verzichtet. Die Hexonbasen wurden nach der in der voraufgehenden Abhandlung beschriebenen Methode dargestellt, hierbei erwies es sich als unbedingt nothwendig, zur Fällung des Argininsilbers Barythydrat anzuwenden, da unter den Verdauungsprodukten eine Substanz vorhanden ist, welche die Abscheidung des Argininsilbers durch Natronhydrat verhindert. Die Fällung des Histidins durch Quecksilberchlorid wurde in der durch Baryt von Schwefelsäure befreiten Lösung vorgenommen, nachdem vorher Kohlensäure hindurchgeleitet war. Zugleich mit dem Histidin 
fallen die Nucleinbassen, die ebenfalls unlösliche Quecksilberchlorid-Verbindungen geben: Diese können aber nach Entfernung des Quecksilbers mit Schwefelwasserstoff vermöge ihrer geringeren Löslichkeit unschwer vom Histidin getrennt werden.

Das Lysin wurde aus dem Filtrat vom Argininsilber mit Phosphorwolframsäure ausgefällt und zur Analyse in das Pikrat übergeführt.

Der Nachweis des Histidins wurde durch Feststellung der Krystallform und des Schmelzpunktes geführt.

Zum Beispiel wurden in einem Falle aus 5 gr. Sturinsulfat nach zwölfstündiger Trypsindigestion $0,1-0,2$ gr. gut ausgebildete Krystalle von Histidinchlorhydrat mit dem Schmelzpunkt $251-252^{\circ}$ erhalten.

Vom Arginin stellten wir sowohl das saure wie das basische Silbersalz dar. Die Analyse des ersteren ergab folgende Werthe:

0,2274 gr. Substanz gaben $40 \mathrm{ccm}$. Stickstoff bei $12^{\circ}$ und $741 \mathrm{~mm}$. Bar. Berechnet für
Gefunden
N: 20,52
$\mathrm{C}_{6} \mathrm{H}_{14} \mathrm{~N}_{4} \mathrm{O}_{8}, \mathrm{HNO}_{8}+\mathrm{AgNO}_{3}$ 20,64

Das basische Silbersalz ergab bei der Analyse:

1. 0,2290 gr. Substanz gab 0,1694 gr. $\mathrm{CO}_{2}$ und 0,0870 gr. $\mathrm{H}_{2} \mathrm{O}$.

2. $0,2249 \mathrm{gr}$. Substanz gab $37,4 \mathrm{ccm} . \mathrm{N}_{2}$ bei $13^{\circ}$ und $760 \mathrm{~mm}$. Bar.

3. 0,2603 gr. Substanz gab 0,1031 gr. AgCl.

$\begin{array}{lcc} & \text { Gefunden } & \mathrm{C}_{6} \mathrm{H}_{14} \mathrm{~N}_{4} \mathrm{O}_{2}+\mathrm{AgNO}_{3}+1 / 2 \mathrm{H}_{2} \mathrm{O} \\ \mathrm{C} & 20,17 & 20,40 \\ \mathrm{H} & 4,22 & 4,25 \\ \mathrm{~N} & 19,66 & 19,83 \\ \mathrm{Ag} & 29,81 & 30,59\end{array}$

Das Lysin wurde als Pikrat dargestellt und analysirt.

0,1532 gr. des zweimal umkrystallisirten Salzes lieferte $24,5 \mathrm{ccm}$. Stickstoff bei $12^{\circ}$ und $745 \mathrm{~mm}$. Bar.
$\mathrm{N}: \quad \begin{gathered}\text { Gefunden } \\ 18,60\end{gathered}$
Berechnet für $\mathrm{C}_{6} \mathrm{H}_{14} \mathrm{~N}_{8} \mathrm{O}_{2}, \mathrm{C}_{6} \mathrm{H}_{7} \mathrm{~N}_{8} \mathrm{O}_{7}$ 18,67

Wie schon oben erwähnt, erhielten wir bei einem andern Versuch ein bisher nicht beschriebenes Silbersalz. Dasselbe wurde folgendermassen dargestellt. 
15 gr. Sturinsulfat wurden unter reichlichem Zusatz von Chloroform einer 12 stündigen Trypsinverdauung unterworfen und sodann in früher beschriebener Weise durch Quecksilberchlorid ausgefällt. Die vom Quecksilberniederschlag abfiltrirte Flüssigkeit wurde mit Phosphorwolframsäure ausgefällt, der gut ausgewaschene Niederschlag mit Baryt zerlegt und der überschüssige Baryt durch die genau entsprechende Menge Schwefelsäure entfernt. Der Rückstand wurde nach dem Eindampfen mit Salpetersäure bis zur schwach sauren Reaction versetzt und nach Zusatz von Silbernitrat mit Alkohol gefällt. Hierbei krystallisirte vorwiegend Argininsilbernitrat aus. Die abfiltrirte alkoholische Lösung ergab auf Zusatz von Aether von Neuem ein in weissen Nadeln krystallisirendes Silbersalz, dessen Analyse Folgendes ergab:

1. 0,2278 gr. Substanz gab 0,1744 gr. $\mathrm{CO}_{2}$ und 0,0854 gr. $\mathrm{H}_{2} \mathrm{O}$.

2. 0,2146 gr. Substanz gab $32,8 \mathrm{ccm}$. Stickstoff bei $12,0^{0}$ und $761 \mathrm{~mm}$. Bar.

3. 0,1872 gr. Substanz gab $28,7 \mathrm{ccm}$. Stickstoff bei $14^{0}$ und $764 \mathrm{~mm}$. Bar.

4. $0,2394 \mathrm{gr}$. Substanz gab $0,0649 \mathrm{gr}$. AgCl.

5. 0,3334 gr. Substanz gab 0,0906 gr. AgCl.

\begin{tabular}{|c|c|c|}
\hline & Gefunden & $\begin{array}{c}\text { Berechnet für } \\
\mathrm{C}_{18} \mathrm{H}_{38} \mathrm{~N}_{7} \mathrm{O}_{5}, 4 \mathrm{HNO}_{3}+2 \mathrm{AgNO}_{3}\end{array}$ \\
\hline $\mathrm{G}$ & 20,87 & 21,15 \\
\hline & 4,16 & 3,82 \\
\hline & $18,12 \quad 18,13$ & 17,82 \\
\hline & $20,80 \quad 20,74$ & 21,15 \\
\hline
\end{tabular}

Die berechnete Formel $\mathrm{C}_{18} \mathrm{H}_{3 \bar{j}} \mathrm{~N}_{7} \mathrm{O}_{5}$ lässt sich leicht mit den bisher beobachteten Erscheinungen in Zusammenhang bringen. Sie kann in folgender Weise aufgelöst werden:

$$
\mathrm{C}_{18} \mathrm{H}_{95} \mathrm{~N}_{7} \mathrm{O}_{5}+\mathrm{H}_{2} \mathrm{O}=\mathrm{C}_{6} \mathrm{H}_{9} \mathrm{~N}_{3} \mathrm{O}_{2}+2 \mathrm{C}_{6} \mathrm{H}_{14} \mathrm{~N}_{2} \mathrm{O}_{2} \text {. }
$$

Dies würde der Zusammensetzung aus $1 \mathrm{Mol}$. Histidin und $2 \mathrm{Mol}$. Lysin entsprechen. Man wird zu der Vorstellung geführt, dass in dem Körper $\mathrm{C}_{18} \mathrm{H}_{35} \mathrm{~N}_{7} \mathrm{O}_{5}$ ein Zwischenprodukt vorliegt, welches nach Abspaltung des Arginins aus dem Sturin hervorgeht und aus 3 Molekülen Hexon zusammengefügt ist, ein «Hexotrion». Leider war die Substanzmenge zu gering, um diese Vermuthung experimentell zu begründen. 
Es ergibt sich aus dieser Beobachtung eine Anregung zu neuen Untersuchungen, welche weitere Aufschlüsse über den physiologisch so wichtigen Zusammenhang zwischen Eiweiss und Kohlehydrat versprechen.

Nach diesen Ergebnissen erscheint die Wirkung des Trypsins auf die Protamine als ein Analogon der Wirkung diastatischer Fermente. Ebenso wie die Letzteren die Polysaccharide in Hexosen und zum Theil auch in Hexobiosen zerlegen, erfolgt unter der Wirkung des Trypsins eine Aufspaltung der einfachsten Eiweissstoffe, indem Hexone gebildet werden. Bei den complicirteren Eiweisssubstanzen - den Eiweisskörpern im gewöhnlichen Sinne des Worts - ist die Wirkung. die gleiche, doch werden hier zugleich mit der Sprengung des Protaminkerns noch Leucin, Tyrosin, Asparaginsäure und andere Atomgruppen frei. Man könnte dieser Anschauung das Bedenken gegenüberstellen, dass diese Spaltung doch keine vollkommene sei, da nach Kühne bei der Trypsinverdauung des Eiweisses ein grösserer Atomcomplex, die "Antigruppe», erhalten bleiben soll. Zur Heurtheilung dieses Einwandes sei auf die folgenden Untersuchungen des Herrn Dr. Kutscher verwiesen. 